\title{
GPs' perceptions of the management of ADHD in primary care: a study of Wandsworth GPs
}

Nicola Salt, Edward Parkes and Amy Scammell Battersea Research Group, Bolingbroke Hospital, Wakehurst Road, London, UK

Attention-deficit hyperactivity disorder (ADHD) is a growing diagnosis in child mental health in the UK and is increasingly being treated with methylphenidate (Ritalin, Equasym, Concerta). There are, however, clinical and public controversies over the diagnosis and 'labelling' of ADHD as a disorder, the use of drug treatments, and a relative paucity of guidance on the initial diagnosis and referral of the disorder. General practitioners (GPs) are involved in the assessment, diagnosis and treatment of children with $A D H D$ and in liasing with the other parties involved, such as parents, teachers and specialists. Therefore their understanding of ADHD and its treatment is important. This study explores Wandsworth (South West London) GPs' understanding of ADHD as a disorder and their views of its management in order to provide more precise detail about the issues concerning the management of ADHD in primary care by GPs.

Qualitative information was collected by semi-structured interviews (with 13 GPs) and quantitative information by means of questionnaires (93 completed by GPs) in Wandsworth.

Whilst GPs had differing views of the aetiology of ADHD, there was a consensus view about the division of responsibility in the treatment of those diagnosed with ADHD. GPs felt uncomfortable initiating the prescribing of methylphenidate and stressed the importance of ongoing specialist involvement in the management of ADHD. There was also a feeling of inadequacy in terms of the training that GPs had received.

It is suggested that guidance on the initial diagnosis of ADHD is drafted for GPs and that shared care protocols are agreed between primary care and secondary care so that the ongoing division of labour in the management of ADHD is made explicit, ensuring continuity of care.

Key words: attention-deficit hyperactivity disorder; attitudes and opinions; general practitioners; methylphenidate; primary care; secondary care

\section{Introduction}

Attention-deficit hyperactivity disorder (ADHD) is an increasingly common diagnosis in child and adolescent mental health. The prevalence of ADHD has risen most sharply in the USA but in England the current prevalence is now estimated at

Address for correspondence: Amy Scammell, Battersea Research Group, Bolingbroke Hospital, Wakehurst Road, London SW11 6HN, UK. between $1.4 \%$ and $6 \%$ (Meltzer et al., 2000). One of the most commonly used diagnostic criteria in the identification of ADHD are those of the Diagnostic and Statistical Manual of mental disorder IV (DSM IV) (American Psychiatric Association, 1994), where six or more behaviours/symptoms of inattention, hyperactivity and impulsivity must have persisted for at least six months and be judged to be maladaptive in order for a child to be diagnosed with the disorder. There is a range of treatments for ADHD from psychological and behavioural therapies (such as behavioural or 
family therapy) to licensed stimulant medications, the most common of which is methylphenidate (Ritalin) (Williams et al., 1999). However, the validity of ADHD as a diagnostic category and the appropriateness of its treatment with stimulants is viewed as controversial by some (Jensen, 2000; DeGrandpre, 2000; Wolraich, 1999).

Traditionally in the UK, the treatment of ADHD has been managed predominantly in secondary care. However, this responsibility has recently shifted towards community and primary care (Thapar and Thapar, 2003) and GPs are now important 'gate-keepers' to treatment for those who might suffer from the disorder. Whilst in other countries there is guidance for primary care professionals on the diagnosis and management of ADHD (Homer et al., 2000; Perrin et al., 2001), in the UK the published guidance does not fully elaborate the role of GPs (National Institute for Clinical Excellence, 2000; Scottish Intercollegiate Guidelines Network, 2000; South West London \& St George's Mental Health NHS Trust, 2002), and published protocols on initial investigations of the disorder are aimed at secondary care. Given that Bramble (2003) reports that between 1995 and 2000 there was a 10-fold increase in the rate of prescribing of methylphenidate in community situations, clarity over management and treatment of ADHD by GPs is essential.

When it comes to research into the management of ADHD there is a relative paucity of studies of primary care in comparison to those concerning secondary care (e.g., Kwasman et al., 1995; Sayal and Taylor, 1997; Lobar et al., 1999) or even concerning its identification in education (e.g., Brook et al., 2000; Sciutto et al., 2000). What little primary care focused research there is comes mainly from outside the UK, which limits its applicability to the UK context, and has tended to centre, directly or indirectly, on stimulant prescribing rather than on wider primary care management (Wolraich et al., 1990; Eppright et al., 1998; Jensen et al., 1999; Angold et al.,2000; Shaw et al.,2002). The only UK survey of GPs was conducted by Ball (2000) in Wales, which presented a consensus of opinion amongst respondents regarding drug treatments for ADHD. Here a majority of respondents reported that these should be initiated by a specialist who would continue to provide clinical monitoring, but many felt that GPs could provide ongoing prescribing and physical monitoring. The study also highlighted GPs perceived lack of training in this area, an element that is mirrored by Shaw and colleagues findings (Shaw et al., 2002; 2003) amongst Australian GPs. Previous studies also tend towards quantitative methods when researching ADHD in primary care either through surveys of professionals or by assessing patients with diagnostic tools and comparing this to the diagnosis they received from their family practitioner, or a combination of both. As a consequence of these methodologies the specific details of the primary care management of ADHD have not been tackled and may more easily be explored by qualitative methods. Klasen (2000) and Klasen and Goodman (2000) both report qualitative methodology in research on ADHD and primary care conducted in the UK. In these studies the focus was on highlighting the differences in the conceptualizations of the disorder between GPs and parents, the dynamics between the two parties and the motivations for a diagnosis of ADHD in each party rather than exploring the wider issues in management. In this respect, the intricacies of GPs understandings of ADHD and how these relate to treatment and management have yet to be fully explored.

This paper reports on research that elaborates on the issues that UK GPs face in the management of ADHD in primary care. It is crucial to have more evidence in this area in a British context, as there are significant differences between international health care systems. This study was based in Wandsworth, South West London and aimed to explore local GPs' conceptions of ADHD and its treatment and management in primary care. To reflect the aims of the research, the study utilizes both quantitative and qualitative methods to provide a broader view of the issues in primary care. It has not sought to engage in the debate concerning over- or under-diagnosis of ADHD, or over- or under-use of methylphenidate. Instead it aims to make useful recommendations on improving the ability of primary care to deal with ADHD in an effective and confident manner.

\section{Method}

The research comprised two parts: a) a questionnaire survey sent to all GPs in Wandsworth Primary Care Trust and b) interviews with GPs working in the Battersea locality of Wandsworth. 
Ethical approval was obtained from Wandsworth Local Research Ethics Committee.

\section{Questionnaire survey}

A questionnaire was designed to elicit Wandsworth GPs' perceptions and understandings of ADHD. This was piloted before being sent out to the full sample. The questionnaire covered the following areas:
a) demographic characteristics of the practitioner respondent;
b) influences on the development of ADHD;
c) symptoms and diagnosis;
d) specialist referrals;
e) shared care;
f) attitudes towards ADHD.

The sample frame was a list of 179 GPs obtained from the locality offices of Wandsworth Primary Care Trust. Questionnaires were sent out in two waves over a two-month period to maximize the response. A letter signed by the lead researcher (who is a local GP) was included with the questionnaire and questionnaires were returned in reply-paid envelopes to the lead researcher at the Battersea Research Group offices. The data was analysed using the statistics package SPSS (Statistical Package for the Social Sciences, V.10).

Fifty-three per cent (98) of the questionnaires were returned, five of which were excluded from the analysis (three were returned blank and two of the addressees had moved away) leaving a sample size of 93 . Rather more $(60 \%)$ of the respondents were female than male reflecting the sample frame.

\section{Interviews}

An interview topic guide was created by the researchers on the basis of key issues identified from the literature to explore the concerns of local GPs. It covered the following areas:

a) GPs' presonal beliefs about ADHD (including its causal factors);

b) their confidence in recognizing and making a formal diagnosis of the disorder;

c) their referral procedures;

d) previous training in the area;

e) their clinical experience of managing patients with ADHD; f) their knowledge of, and attitudes towards, the various treatments of ADHD;

g) their specific feelings regarding the prescribing of methylphenidate.

A convenience sampling method was used to recruit GPs into this part of the study and 13 GPs from within the Battersea locality were interviewed. Seven were male and five were female and the average age was 41.3 years (S.D., 9.3). Each interview lasted approximately half an hour and two researchers conducted the 13 interviews between them. The recordings of the interviews were transcribed and these transcripts were analysed using the computer package NVivo (QSR International). Qualitative summaries of issues raised by the GPs in each section of the interview schedule are presented.

\section{Results}

\section{Questionnaire survey}

The questionnaire items were analysed to look at high consensus items to highlight the level of agreement between GPs on each topic.

\section{Influences on the development of $A D H D$}

The respondents' understandings of the influences on the development of ADHD were mixed (see Figure 1). The factors believed to influence ADHD the most were genetic, chemical imbalance and quality of parenting. Those least likely to influence ADHD were reported as social class, peer group and ethnicity.

\section{Symptoms of $A D H D$}

It is possible to see from Figure 2 that most GPs included the three diagnostic criteria from the DSM (impulsivity, inattention and hyperactivity) when listing symptoms of ADHD. However, over $75 \%$ of GPs thought 'educational underachievement', 'antisocial behaviour' and 'sleep problems' were also symptoms of the disorder despite their non-inclusion in standard DSM diagnostic criteria. Only three GPs restricted themselves to just three symptoms/behaviours.

\section{Referral for treatment}

Sixty-nine per cent of respondents indicated that they would refer a patient with ADHD to a child 


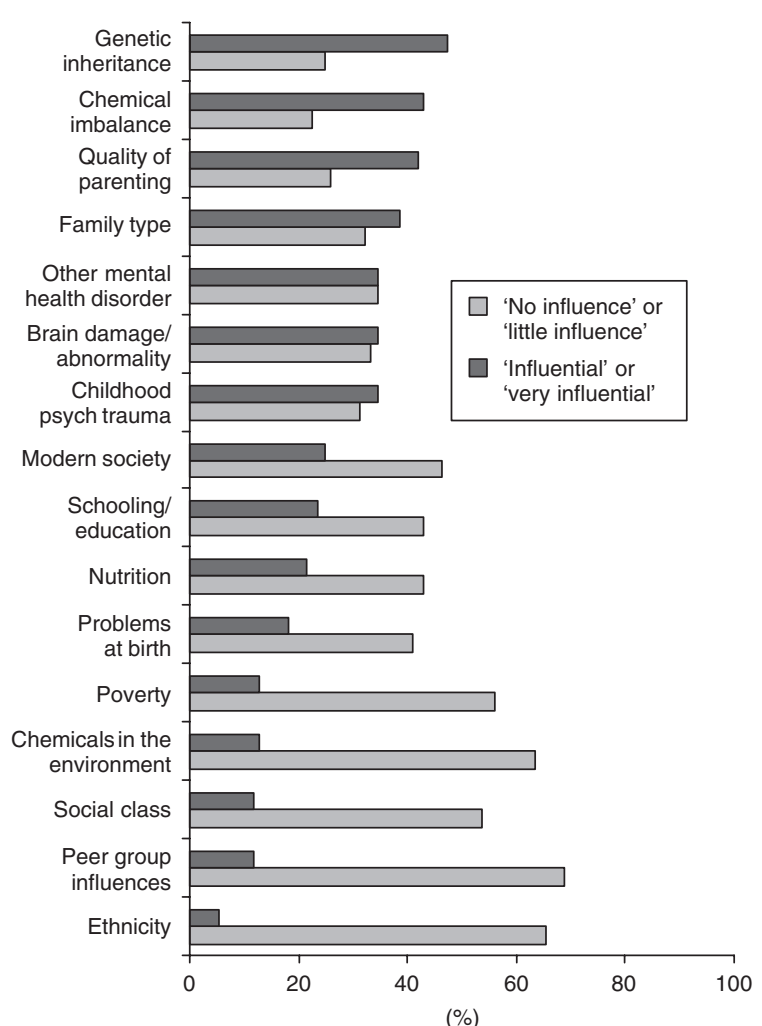

Figure 1 Influences on the development of ADHD. Percentage of respondents who thought the following list of factors influenced the development of ADHD. The percentages for the two extreme response categories ('influential' and 'very influential, 'no influence' and 'little influence') have been collapsed and the middle response ('somewhat influential') is omitted. $n=93$.

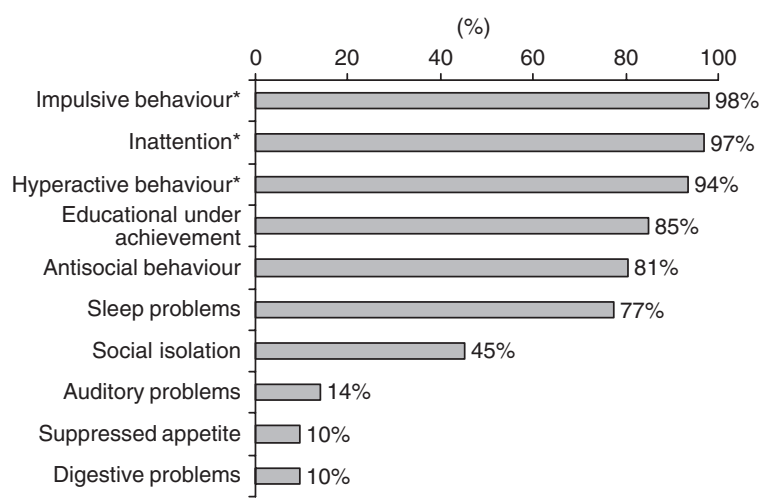

Figure 2 Symptoms of ADHD. Percentage of respondents who thought the indications given in the figure were symptomatic of ADHD. $n=93$. *: DSM IV diagnostic criteria.

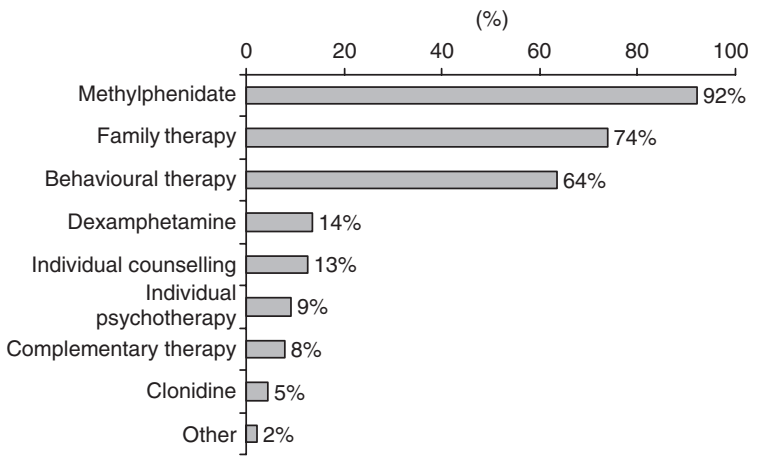

Figure 3 Treatments for ADHD. Percentage of respondents who thought the indications given in the figure were effective treatments for ADHD. $n=93$.

psychiatrist, followed by $28 \%$ who would refer to a paediatrician, $15 \%$ to an educational psychologist, $10 \%$ to a clinical psychologist, $2 \%$ to a psychotherapist and $1 \%$ to a counsellor or to another specialist. Seventeen per cent of respondents indicated more than one specialist to whom they would refer.

\section{Types of treatment}

Overall, almost $90 \%$ of respondents indicated more than one treatment that they thought was effective and the number of those who indicated each treatment can be seen in Figure 3. Methylphenidate was the most common treatment $(92 \%)$, followed by family therapy and behavioural therapy. Twelve respondents did not think that methylphenidate was the most effective treatment for ADHD and one of these suggested another pharmacological treatment in its place.

\section{Aspects of shared care}

In relation to the management between the GP and specialist of the treatment of the patient, the shape of shared care in the eyes of GPs seemed consistent (see Table 1). They preferred to let the specialist have primary responsibility for the investigation and diagnosis of the disorder and also for the initial prescribing of methylphenidate. The majority of GPs were happy to take responsibility for repeat prescribing. However, the majority of GPs felt that the specialist should have primary responsibility for the ongoing monitoring of patients although almost $35 \%$ thought that the GP, or the GP in combination with the specialist, could fulfil this role. 
Table 1 Aspects of shared care. Individual with primary responsibility for the management of various aspects of ADHD. Percentage of respondents (majority response in bold). $n=93$.

\begin{tabular}{llllllll}
\hline & Specialist & GP & Other & $\begin{array}{l}\text { Specialist } \\
\text { and GP }\end{array}$ & $\begin{array}{l}\text { Specialist } \\
\text { and other }\end{array}$ & $\begin{array}{l}\text { GP and } \\
\text { other }\end{array}$ & All \\
\hline Initial investigations & $\mathbf{7 5}$ & 19 & 0 & 4 & 0 & 0 & 0 \\
Formal diagnosis & $\mathbf{9 6}$ & 1 & 1 & 0 & 1 & 0 & 0 \\
Initial prescribing & $\mathbf{9 7}$ & 0 & 0 & 0 & 0 & 0 & 0 \\
Repeat prescribing & 21 & $\mathbf{7 3}$ & 0 & 3 & 0 & 0 & 1 \\
Patient monitoring & $\mathbf{6 0}$ & 14 & 0 & 20 & 0 & 1 & 1 \\
\hline
\end{tabular}

\section{Attitudes towards ADHD}

GPs attitudes to ADHD are summarized in Figure 4. GPs cited strongest agreement with the statement that the disorder could lead to the patient being stigmatized and strongest disagreement with the statement that ADHD is uncontroversial. On the whole, GPs thought that the media had a significant effect on the public's conception of the disorder, that a diagnosis of the disorder could lead to the patient being stigmatized and that the disorder was not solely one of childhood. There was more variation in attitudes over how well defined a disorder ADHD is and its treatment with drugs.

\section{Interviews}

GPs' understanding and conceptions of $A D H D$

The interviews confirmed questionnaire findings to the extent that GPs offered different definitions of ADHD but most of these included the three criteria of inattention, hyperactivity and impulsiveness of the DSM IV diagnosis (American Psychiatric Association, 1994). However, GPs' confidence in their conceptions varied.

A child's behaviour was defined as problematic to the extent that it affected others. GPs tended to focus on inattention and poor concentration, especially when in school. This aspect was especially salient in the regular comment that children could not 'sit still':

Well for me its (ADHD) when you have a child who is extremely inattentive, does not comply with instructions and is very disruptive, and when amongst his peers tends to distract everybody. He doesn't sit down normally, he has to be doing something or wanting to be doing something whilst the others are concentrating. If the mother brings the child with that particular problem then ADHD comes into my mind.

(GP 3)

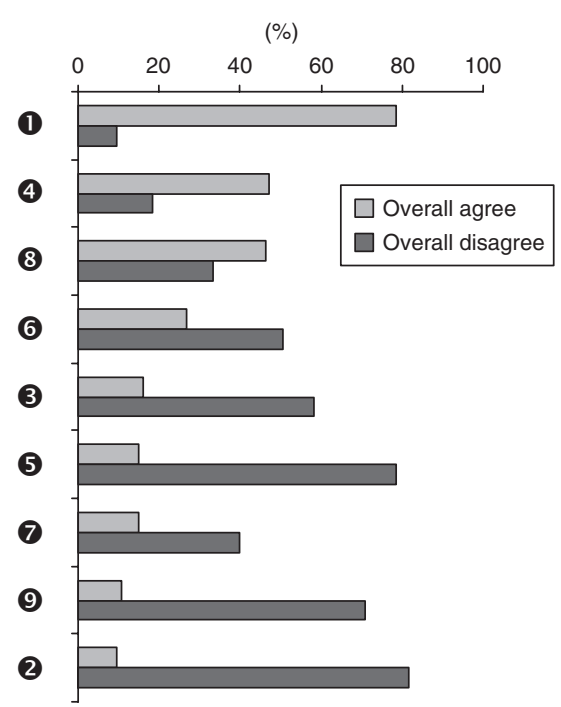

1. Patients can be stigmatized and disadvantaged by the diagnosis of ADHD;

2. ADHD is an uncontroversial disorder;

3. One should avoid treating ADHD with drugs;

4. Parents have a vested interest in having their children being diagnosed with ADHD (e.g., it shifts blame);

5. The media has little effect on people's conception of ADHD;

6. ADHD is a well-defined psychiatric disorder;

7. Successful drug treatment proves that ADHD is a biological disorder;

8. It is hard to know which type of behaviour is age appropriate and which is due to ADHD;

9. ADHD only exists in children.

Figure 4 Attitudes towards ADHD. Percentage responding to each attitude item. $n=93$.

Basically it is a case which I have seen sometimes in the past where behaviour seems to be the biggest problem ... behaviour in any organisation, behaviour at home, at school, or in any nurseries. People find it difficult to 
either communicate with these kids or to listen to them and what they're asking for.

(GP 12)

As far as causes of ADHD were concerned, all GPs were either unsure or stated that the true cause was still unknown:

The cause is not known, I don't think it's known. I think it's the action of a genetic switch or mutant somewhere, but I don't think I know the cause.

(GP 5)

However, most offered some sort of explanation of causes. Causation was seen by GPs as being multi-factorial with both biological and social components. However, a statement about the multifactorial nature of causation was usually accompanied by statements about the GP's own beliefs on key influences:

I'm really split between the biochemical thing and Ritalin and parenting. I think it's probably going to be a combination of both, like depression is a combination of biochemical problems usually secondary to life events, isn't it? But I think there's an environmental thing as well. Saying its purely genetic is a bit of a cop out; your don't have to point fingers at anybody, it makes life a lot easier.

(GP 9)

GPs also highlighted their awareness of controversy:

I think that there is a lot of argument over this but I think that it is behavioural, parenting causing a lot of it, but I don't know whether that's necessarily what's diagnosed as ADHD... there's a lot of controversy at the moment.

(GP 7)

All GPs were uncertain about the precise rate of diagnosis in this country. Over half of GPs thought that the disorder was under-diagnosed whereas the others either did not know, thought it was overdiagnosed or that the level was dependent on the precise circumstances being discussed. The GPs cited varying reasons for their answers. The divided opinions surrounding the disorder, as well as the difficulty in its diagnosis, were cited by GPs who did not know the level of diagnosis for ADHD as well as those who thought it was both over- and underdiagnosed. Those who thought that ADHD was under-diagnosed justified this through comparison with the prevalence of the disorder with the USA:

I would say at this time as a condition it's probably under-diagnosed. Because I mean I'm thinking about the amount I see in a list of 2000, I've got one child. I mean compared to America, where it's much higher ... It depends where you compare it to ... Depends whether you're comparing it generally, or comparing it to America.

(GP 10)

In their justifications, those who thought it was under-diagnosed also cited the fact that the disorder was relatively newly defined and that GPs did not have adequate training in its recognition:

Basically it's a new diagnosis. It's been identified for some time but it's quite fashionable now, along with dyslexia and the rest.

(GP 4)

It's like a lot of illnesses, certainly when I was at medical school, which was a long time ago now, ADHD was not actually even heard of.

(GP 2)

Those GPs who said they did not know the exact prevalence, or that the prevalence was variable, justified this assumption by emphasizing that it was hard to gauge the level of diagnosis:

I don't know what the rates of diagnosis are. And I'm sure that there are those who have it that don't reach the health authorities and because of social construct, there are children in one context who may be diagnosed with it and in another aren't

(GP 11)

\section{Management and experience of $A D H D$}

GPs were asked about their confidence in diagnosing ADHD and over half lacked confidence in recognizing the condition. Most GPs stressed the importance of specialist involvement in the diagnosis:

I think it's a specialist decision and if I suspected, I would want a specialist to confirm it. I would want a specialist to say nine out of ten it's ADHD or three out of ten it's not ADHD. I do not think it's my decision.

(GP 12) 
Most GPs would refer to the child psychiatric/ mental health unit and only one GP was unclear about whom to refer to having never done so. Most GPs would prefer to refer the child to a specialist, but if they did want to confirm their suspicions they knew they had to take a thorough history of the patient and to have confirmation from the parents and the child's school. However, they did not seem to be completely sure about the specific diagnostic procedures - one GP mentioned that they would observe the child in the consultation room and no GPs mentioned any specific ratings scales. The reasons for GPs' reticence about making the diagnosis themselves included the limits of their own training, the negative consequences of labelling and the contentiousness of the treatment. However, it seemed that the actual shape of 'shared care' that GPs were offered, differed from that which they would prefer. In talking about local 'shared care' between primary and specialist services, one GP highlighted problems in collaboration with secondary care, which impacted on patient management:

Basically we got a letter from our local consultant ... [which said they were] handing back all uncomplicated cases of ADHD for us to deal with, even though the drug and therapeutic guidelines have said, and a certificate has been issued saying that initially diagnosis should be by a specialist, and then for routine monitoring they can be handed back. But every year the specialist has to collaborate with the GP, so the patient is still monitored under some specialist care. ... There was not collaboration between primary and secondary care, basically the specialists were just chucking it all back to us and we were left to deal with it ...

(GP 10)

No GP received any undergraduate training concerning ADHD and this was explained in relation to the fact that ADHD is a 'new diagnosis' and was not in their curriculum. Some GPs had read up on the subject and only one had attended any lectures on child and adolescent mental health.

All GPs have had contact with at least one patient with ADHD and one was familiar with half a dozen. The GPs gave examples in which the majority of patients were on Ritalin (methylphenidate) or had been prescribed it in the past. The majority of GPs described their involvement in the repeat prescribing of stimulants:

Well we've got some children here. We don't actually see them very much, we tend to sort of continue prescribing, and then they're followed up by the psychiatrist.

(GP 13)

In describing their experience of patients with ADHD, GPs would often give a vignette in which social factors concerning the child's family background were emphasized:

Right, he's twelve, he's from, what's the word, a very dysfunctional family, he's on Ritalin. He's not eating very well. He goes to a day centre, and he's OK, he's quite hyperactive when he comes in. He walks around the room, starts playing with toys and gets out stuff from cupboards and drawers ...

(GP 10)

I've dealt with one patient with ADHD. He's eight years old and has all the typical clinical symptoms, you know, he can't concentrate on anything, can't sit still, lots of problems at home. The parents are having marital discord I think ... I think that's what the problem is.

All GPs were aware of methylphenidate (Ritalin) as a treatment for ADHD and were also aware of other types of treatment including family therapy and counselling. Most thought that a combined approach was most appropriate in the treatment of ADHD and that both methylphenidate and behavioural techniques were important:

I think the psychiatric diagnosis should always have behavioural support associated with it. It's not just the drug and the doctor, it's the whole situation.

(GP 1)

All of them together is best I think. I think they go hand in hand. Behavioural therapy is useful and Ritalin stabilizes the child.

(GP 13) 
Again the shape of shared care that came across was that GPs were unhappy in initiating the prescribing of methylphenidate and they wanted this done by specialists but that they were happier in continuing its prescribing:

I would not be happy to initiate it. Once the diagnosis has been made by a specialist though then I would be quite happy to initiate it.

(GP 5)

Their contentment with continuing prescribing was reliant to a certain extent on specialists in the follow-up and monitoring of patients:

... you want to see the child every six months because you want to get their weight and blood pressure and that sort of thing. But again you see, that sort of follow-up had been done by the hospital. The hospital tells us what to do and we just do it ...

(GP 9)

Despite this conception of the division of responsibility between specialist and GP, GPs did have their own reservations about the prescribing of methylphenidate:

I suspect Ritalin is prescribed because it is easier than giving counselling. I don't know how effective counselling might be. I'd prefer some kind of counselling myself because I'm inherently suspicious about the use of drugs in children and what it does to their longterm development.

(GP 6)

Several GPs had explicit reservations about continuing to prescribe the drug whereas others were more comfortable with the situation. GPs did not seem concerned about the costs associated with the prescribing of methylphenidate but were worried that patients might not draw the appropriate monitoring from specialists.

Probably in legal terms the liability lies with me but I can cope with that ... Difficulty in liasing with specialists is not a problem I have had, but again I only have one case. Certainly if the specialist was being difficult I would say 'well look I don't want to keep doing this if you're not playing your part'. But I would hope they wouldn't do that.

(GP 2)
In terms of the follow-up checks conducted on children prescribed methylphenidate, GPs appeared to be guided by the specialist, if they were aware of checks at all. As far as specific side effects were concerned, several GPs said there were none or could not remember what they were. Those side effects that were mentioned included fears about dependence, increased aggression and agitation, and insomnia.

\section{Discussion}

Despite a variation in understanding of causes, and to some extent the symptoms, of ADHD, there seems to be a consensus amongst GPs in the sample about the management of ADHD in primary care. Both the interviews and questionnaire survey paint a picture of shared care in which specialists take primary responsibility for the diagnosis of the disorder and also take responsibility for the initial prescribing of methylphenidate in its treatment. GPs were reasonably comfortable in repeat prescribing of methylphenidate but it seemed that they did not have the necessary experience or knowledge to be able to manage the monitoring of children on stimulant treatment. GPs felt ongoing monitoring was a responsibility of the specialist involved and this also came out in the questionnaire analysis.

It is interesting that GPs were willing to consider both biological and social influences on the development of ADHD but that the treatment of the disorder seemed to be mainly psychopharmacological. This might be a reflection of the lack of availability in general practice of other treatments or perhaps a reflection of the guidance that is published on the treatment of the disorder. It may be that GPs felt unable to question the treatment given to patients due to their lack of knowledge of the subject, which itself was something they often commented on. A more pressing corollary of GPs' limited knowledge was the fact that they were unclear about the side effects of methylphenidate and the ongoing monitoring required. If the assumption on their behalf is that this would be dealt with by the specialist involved, it could be the case that important signs are missed if there is inadequate communication with the specialist. This would be especially true if specialists assumed a higher level of monitoring of cases by GPs. 
The interviews brought up interesting findings that merit further exploration, such as the relationship between GPs understanding of ADHD and how this impacts on actual treatment. Sayal et al. (2002) from their study suggest that parents have more influence on a child's access to care than GPs so this issue might be inconsequential. However, given the possibility of disempowerment of parents by a lack of a diagnosis (Klasen, 2000), GPs' conceptualization of ADHD should still be given attention. It would also be worthwhile expanding the study to include the specialists with responsibilities for children in Wandsworth to see whether their conceptions of shared care conflict with those of GPs.

\section{Limitations of the study}

This study was an exploratory investigation and did not look at the actual provision of care for those with ADHD by Wandsworth GPs. This would be worthwhile, as would expanding the study to include the specialists with responsibilities for children in Wandsworth to see whether their conceptions of shared care conflict with those of GPs. The results from this study should therefore be generalized with caution. Some results in particular are likely to be specific to the Wandsworth area. For instance, the particular specialist to whom GPs refer is likely to be dependent on local service provision more than anything else. However, the shape of shared care preferred by the GPs, and the wider lessons for GP training, are probably transferable to most services in the UK.

\section{Recommendations}

Given the variety of conceptions of ADHD found, and the controversy that still exists in the literature, we suggest that evidence-based guidance on the initial diagnosis and referral of ADHD for GPs would be helpful. This could include protocols for structured communication between primary and secondary care. However, guidelines on their own may not be enough. In the interview element of the study we found that GPs could be more fully aware of the possible side effects from methylphenidate. This type of lack of information would probably be best tackled through training, something which previous research has also suggested (Ball, 2000; Shaw et al., 2002). Perhaps there is also a need for training for GPs on how to deal with 'incompletely medicalized' (Klasen, 2000) disorders, such as ADHD.

\section{Acknowledgements}

This study was funded by the Battersea Primary Care Group and received ethical approval from Wandsworth Local Research Ethics Committee. We would like to thank Dr Rosie Savage for her help in the development of this study especially the questionnaire and Dr Antonia Bifulco for giving comments on an earlier version of this article. We would also like to thank all those GPs in Wandsworth who gave their time to contribute to this research.

\section{References}

American Psychiatric Association. 1994: Diagnostic and statistical manual of mental disorder $I V$, Washington, DC: American Psychiatric Association.

Angold, A., Erkanli, A., Egger, H.L. and Costello, E.J. 2000: Stimulant treatment for children: a community perspective. Journal of the American Academy of Child and Adolescent Psychiatry 39, 975-84.

Ball, C. 2000: Attention deficit hyperactivity disorder (ADHD) and the use of methylphenidate: a survey of the views of General Practitioners. Psychiatric Bulletin 25, 301-304.

Bramble, D. 2003: Annotation: the use of psychotropic medications in children: a British view. Journal of Child Psychology and Psychiatry 44, 169-79.

Brook, U., Watemberg, N. and Geva, D. 2000: Attitude and knowledge of attention deficit hyperactivity disorder and learning disability among high school teachers. Patient Education and Counseling 40, 247-52.

DeGrandpre, R. 2000: Ritalin nation: rapid-fire culture and the transformation of human consciousness, London: Norton.

Eppright, T.D., Bradley, S., Vogel, S.J. and Williamson, H.A. 1998: The management of patients with attention-deficit hyperactivity disorder by family practitioners. Missouri Medicine 95, 118-22.

Homer, C.J., Blatz, R.D., Hickson, G.B., Miles, P.V., Newman, T.B., Shook, J.E., Zurhellen, W.M., Lowe, B.A., Schwalenstocker, E., Goldberg, M.J., Shiffman, R., Berger, J.E., France, F.L., Perrin, J.M., Stein, M.T., Amler, R.W., Blondis, T.A., Feldman, H.M., Meyer, B.P., Shaywitz, B.A., Wolraich, M.L., Despirito, A., Pierce, K., Ganiats, T.G., Grabert, B. and Brown, R.T. 2000: Clinical practice guideline: diagnosis and evaluation of the child with attention-deficit/hyperactivity disorder. Pediatrics 105, 1158-70. 
Jensen, P.S. 2000: Current concepts and controversies in the diagnosis and treatment of attention deficit hyperactivity disorder. Current Psychiatry Reports 2, 102-109.

Jensen, P.S., Kettle, L., Roper, M.T., Sloan, M.T., Dulcan, M.K., Hoven, C., Bird, H.R., Bauermeister, J.J. and Payne, J.D. 1999: Are stimulants overprescribed? Treatment of ADHD in four U.S. communities. Journal of the American Academy for Child and Adolescent Psychiatry 38, 797-804.

Klasen, H. 2000: A name, what's in a name? The medicalization of hyperactivity, revisited. Harvard Review of Psychiatry 7, 334-44.

Klasen, H. and Goodman, R. 2000: Parents and GPs at crosspurposes over hyperactivity: a qualitative study of possible barriers to treatment. British Journal of General Practice 50, 199-202.

Kwasman, A., Tinsley, B.J. and Lepper, H.S. 1995: Pediatrician knowledge and attitudes concerning diagnosis and treatment of attention deficit and hyperactivity disorders. A national survey approach. Archives of Pediatric and Adolescent Medicine 149, 1211-16.

Lobar, S.L., Oher, L.E., Waechter, M.L. and Phillips, S. 1999: Parents', physicians' and nurse practitioners' perceptions of behaviors associated with attention deficit hyperactivity disorder. Journal of the American Academy of Nurse Practioners 11, 237-42.

Meltzer, H., Gatward, R., Goodman, R. and Ford, T. 2000: Mental health of children and adolescents in Great Britain. London: The Stationery Office.

National Institute for Clinical Excellence. 2000: Guidance on the use of methylphenidate (Ritalin, Equasym) for attention deficit/hyperactivity disorder (ADHD) in childhood, London: National Institute for Clinical Evidence.

Perrin, J.M., Stein, M.T., Amler, R.W., Blondis, T.A., Feldman, H., Meyer, B.P., Shaywitz, B.A., Wolraich, M.L., Despirito, A., Homer, C.J., Wender, E., Brown, R.T., Ganiats, T.G., Grabert, B., Pierce, K., Herrerias, C.T., Baltz, R.D., Hickson, G.B., Miles, P.V., Newman, T.B., Shook, J.E., Zurhellen, W.M., Lowe, B.A., Schwalenstocker, E., Goldberg, M.J., Shiffman, R., Berger, J.E. and France, F.L. 2001: Clinical practice guideline: treatment of the school-aged child with attention-deficit/hyperactivity disorder. Pediatrics 108, 1033-1044.

Sayal, K. and Taylor, E. 1997: Drug treatment in attention deficit disorder: a survey of professional consensus. Psychiatric Bulletin 21,398-400.

Sayal, K., Taylor, E., Beecham, J. and Byrne, P. 2002: Pathways to care in children at risk of attention-deficit hyperactivity disorder. British Journal of Psychiatry 181, 43-48.

Sciutto, M.J., Terjesen, M.D. and Bender-Frank, A.S. 2000: Teachers' knowledge and misperceptions of attention-deficit/ hyperactivity disorder. Psychology in the Schools 37, 115-22.

Scottish Intercollegiate Guidelines Network. 2001: Attention deficit and hyperkinetic disorders in children and young people. SIGN. Online at http://www.sign.ac.uk (retrieved 15 December 2004).

Shaw, K.A., Mitchell, G.K., Wagner, I.J. and Eastwood, H.L. 2002: Attitudes and practices of general practitioners in the diagnosis and management of attention-deficit/hyperactivity disorder. Journal of Paediatrics and Child Health 38, 481-86.

Shaw, K., Wagner, I., Eastwood, H. and Mitchell, G. 2003: A qualitative study of Australian GPs' attitudes and practices in the diagnosis and management of attention-deficit/ hyperactivity disorder (ADHD). Family Practice 20, 129-34.

South West London \& St. George's Mental Health Trust. 2002: Shared care guidelines for the use of Concerta XL (prolonged release methylphenidate), London: South West London \& St. George's Mental Health Trust.

Thapar, A.J. and Thapar, A. 2003: Attention-deficit hyperactivity disorder. British Journal of General Practice 53, 225-30.

Williams, C., Wright, B. and Partridge, I. 1999: Attention deficity hyperactivity disorder - a review. British Journal of General Practice 49, 563-71.

Wolraich, M.L. 1999: Attention deficit hyperactivity disorder: the most studied and yet most controversial diagnosis. Mental Retardation \& Developmental Disabilities Research Reviews 5, 163-68.

Wolraich, M.L., Lindgren, S., Stromquist, A., Milich, R., Davis, C. and Watson, D. 1990: Stimulant medication use by primary care physicians in the treatment of attention deficit hyperactivity disorder. Pediatrics $86,95-101$. 